\title{
Why a multidisciplinary agenda for Southern Europe?
}

\author{
Teresa de Noronha ${ }^{1}$, Eric $\operatorname{Vaz}^{2}$ \\ ${ }^{1}$ University of Algarve, Faro, Portugal \\ ${ }^{2}$ Ryerson University, Toronto, Canada
}

Received: 18 September 2019/Accepted: 18 September 2019

Since the process of Southern Europe's integration in the European Union, the Mediterranean region has seen a more considerable gap between central and northern European countries and its Southern European counterpart. Thus, in a European context of social cohesion, it becomes necessary to better understand Southern Europe, without escaping to the common perception of the complexity of Mediterranean culture. As a significant player throughout history, Southern Europe consistently established a platform of diversity and freedom, bringing peace between different historic-cultural traditions. Moreover, the southern frontier of Europe to Africa and Asia has become a crucial determinant in the current times of change. Where ruptures in the political systems are also defining new patterns of regional migration. Meanwhile, the integration of Italy, Portugal, Spain, and Greece into the European Union reinforced an essential search for stability, altering to some extent the political and economic predispositions of these countries. This has been followed by somewhat rigid institutions, that remain, to a certain extent, an obstacle to sustainable development, and justify a broader assessment of the potential of policy and governance intervention. In the Mediterranean region stagnation, or increasing poverty, and migration is leading the most impoverished areas to a deleterious deprivation of human resources and capital. Southern European countries may represent a bridging alternative and an exemplar representation of democracy. A positive Mediterranean agenda is necessary, where migration patterns become a substantial factor in the future of all the frontier countries: Italy and Greece, Spain, and Portugal. This special issue collects recent insights in socio-economic developments in Mediterranean countries in order to further a future agenda for Southern Europe.

The future agenda for Southern Europe is multidimensional and requires a multidisciplary approach. Three main dimensions need to be covered by such an agenda: (i) Migrations patterns, whereby sociological and economic aspects take into account the diverse territorial areas and individual regions; (ii) Sustainable economic transitions that cater to better and more integrative business structures; (iii) Entrepreneurship and creativity, where management of scarce natural and financial resources is pursued to suitably create new productive and networking systems, which actively absorb diversified populations in a multicultural and pluralist Europe.

The proposals of the Action Plan on the integration of third-country nationals (European Commission 2016) reinforces the existing "evolutional process". This includes the need for integration policies directed towards local characteristics that promote access to services, education, and jobs. Southern Europe, in the Mediterranean context, is part of an extended effort to discuss, for the first time, this pluralistic agenda. It outlines the complex aspects of southern Europe, which require a significant reflection about the Mediterranean region and the European countries within it. Notably, the region's cultural and populational background. In a forthcoming monograph titled Southern 
Europe - Major Trends and New Prospects (Vaz, de Noronha 2020), the authors Eric Vaz and Teresa de Noronha unravel the complexity of southern Europe's integration from a regional science perspective and discuss these issues from a geographical and economic viewpoint.

Driven by the interest triggered through this contribution, the authors organized a major international conference, entitled "Mediterranean Cultures and Societies" in 2017, at the Research Centre of Spatial and Organizational Dynamics, University of the Algarve, Portugal, in partnership with the ICSR Mediterranean Knowledge, Italy. The high quality of presented papers became a cradle for a broader spectrum of different scholarly contributions within the socio-economic problems of Southern Europe. These contributions have led to the following publication outputs:

1. Historical Contexts and Identities of the Mediterranean Countries, published in the Journal of Spatial and Organizational Dynamics (de Noronha, Mangone 2018), is a collection of eight papers discussing, from a geographical and sociological perspective, case studies related to Mediterranean identities in Southern Europe.

2. The Mediterranean and Migrations, in the Journal of Mediterranean Knowledge (de Noronha 2018), advances some historical and sociological theoretical considerations of what remains one of the greatest challenges of the 21st century for Mediterranean and Europe -migration.

3. Finally, this publication, Southern Europe in the Mediterranean Context in REGION, calls for multidisciplinary aspects suggesting that regional advances in development and sustainability of the region can only occur if a multidisciplinary approach is used.

One of the primary goals of this recently developed research is to call awareness to the need for increasing proximity between the Mediterranean countries and the rest of Europe. The distance grew through the modernization process of the 1950 s and the post-colonial policies of the last century. This process was, at least partially, responsible for the communities integrating external forms of modernity without adding new developments in the process of growth and economic valorisation.

Meanwhile, over the last three decades, the European Union had a significant role in shaping the decision-making processes on economic growth and development across all its member states. However, a growing gap between Northern and Southern Europe has been recognized. Not only did the last international economic recession have negative impacts on Southern European countries but also some profound structural adjustments within Southern European countries occured.

The significant impact of the last economic recession (2008) on sustainable development was felt strongly at a geographical level. While infrastructures built during the nineties still exist in many places, their upkeep and maintenance have become condemned for future generations. Meanwhile, the available policy support and investment in research and development became restricted. This led to some apathy by decision-makers who lacked financial possibilities to invest and maintain the status quo of regions. For example, monitoring initiatives by the European Environmental Agency have shown a paramount increase in urban sprawl in southern Europe, leading to a direct depletion of resources resulting from ineffective planning in the last decades. This takes its toll directly on land use and infrastructure, leaving decision-makers incapacitated to cope with the current crisis.

Surprisingly, and out of a context of adversity, in Portugal and Spain conditions for economic growth and remarkably creative solutions for sustainability are emerging . This shows Southern Europe's resilience, the capacity of innovation, and its historical driving force to face challenges and find new paths to growth and prosperity. From its struggle, many socio-economic examples may be observed, and its heritage and challenges should motivate scholars to further regional research on its challenges.

The objective of this publication is to emphasize a continuous stream of research topics related to the complexity of Southern Europe's economic structures, urban and climate change impacts, urban sprawl and natural heritage across Portugal, Spain, Italy, and 
Greece. We are optimistic that it is through regional science and location-based research that a unique laboratory is formed that may offer innovative solutions to address complex change in national governance post-crises. We are delighted to bring this paradigm to Southern Europe. Many examples of resilience of territories could be added, and remarkable findings on the history of adaptation and economic growth.

This editorial justifies the need to tackle the current situation in Southern Europe from a multidisciplinary perspective. The ubiquitous nature of regional and urban planning has led to the development of a series of instruments and frameworks, embodied in the local policy agendas of Southern European countries. The tools available and digital instruments such as land use and land cover assessments have become crucial instruments to monitor the inevitable change of the rapidly transitioning environments. It is in the reinvention of public space and landscape that the articulation of urban fabric must take place to offer an integrative vision of future sustainability. Such integrative visions call for multidisciplinary actions to understand the context of regions and policy, as well as multi-tiered scales and methods to foster sustainable development of Southern Europe. It is evident that there is still a debate on what is the best approach to improve development in Southern Europe. However, it is through the incorporation of geographical analysis that one can better frame the multidisciplinary actions towards resilience of landscape. Further contributions to an interdisciplinary agenda in Southern Europe enable the efficient detection of the sustainability of urban regions in the South. Thus, leading to proactive action taking advantage of spatial analysis, GIS, and regional science.

The first article in this special issue "Integrated local development in Mediterranean marginal territories: The case study of Casentino (Italy), Algarve (Portugal) and Corse (France)" by Andrea Ricci, Mario Biggeri and Andrea Ferrannini present three case studies across Italy, Portugal, and France to propose lessons learned from a long-term development process at the local level. Today, marginalized Mediterranean territories are facing tremendous challenges but at the same time they have relevant endogenous resources, which are often underutilized and unexploited. In the last decades, they have been characterized by a progressive abandonment in favour of urban areas, with consequent high social costs such as the hydrogeological instability, land degradation, and soil erosion. The paper aims to verify the idea that Mediterranean peripheries, , could take part in the formation of their own development trajectories. Additionally, that these territories can actively contribute to the harmonious development of Europe, creating new jobs opportunities and stable development patterns. The structure of this paper starts with general theoretical arguments and a short description of European policies for development. Then, it follows with a diagnostic analysis of three local territorial contexts - i.e. Casentino (Italy), Algarve (Portugal) and Corse (France). Finally, it returns to the general European issues and proposes implications as well as lessons learnt in the analysis of the development processes at the local level.

The second contribution, "Rehabilitation and Renewal of Mediterranean Structures. The Utopic Landscape of Algarve" by Carlos Bragança dos Santos, explores features of Mediterranean landscapes and their relationship to identity in a context of coastal zones with intense human impact. The author interrelates ecological, aesthetic, symbolic, socio-economic, and political aspects that influence the spatial distribution of structures. Additionally, the author considers/analyzes the image of the terraces as a typical geographic feature of southern European regions. One of the remarkable features of Mediterranean landscapes is the terraced landscape, usually supported by dry stone walling. The terraces, property division walls, pathways, and traditional paths create network compartmentalization that define landscape identity. The informational content, aesthetic quality, ecological, and cultural values allowed by this articulated construction are particularly important in coastal zones with intense human impact. On the Algarve, the hills displayed by such structures are the background to an urban-touristic system. The values that local people may assign to their landscapes will determine the acknowledgment of the structural elements under analysis, but the role of tourists must also be seriously considered. Beyond nostalgic solutions, one must prospect the future of the dry-stone walling structure into the diversity of possible solutions for a sustainable landscape development, which enhances the living part of an inseparable unit that includes 
the densest urbanized areas with less ecological functions.

The third article, "The role of the European Union on immigration. An anthropological approach to the treaties that have been carried out in Europe in order to manage diversity" by Carmen Clara Bravo Torres, brings us to one of the greatest sociological concerns facing Southern Europe recently - migrations. Specifically, this is achieved through an analysis of how EU agreements are not adapted to the emerging migration settings. Migrations are a global phenomenon that has prevailed throughout history. In the last decades, there is a need to control every person who enters and leaves the borders of a country. This fact can be observed in the European Union where, at least, during the last decade, the migratory phenomenon is considered a problem. The EUcarries out different measures to manage this diversity within its borders. However, these measurements are not adapted to the different contexts and are not carried out by all the countries within the EU. Despite all this, the discourse used by the European Comission promotes European identity ahead of the rest, differentiating those who are considered others. These themes are studied in this paper, which will allow readers to understand what treaties have been established in the European Union regarding migration and how national diversity is managed through them.

"The Mediterranean Variety of Capitalism, Flexibility of Work Schedules, and Labour Productivity in Southern Europe" is the fourth article in this special issue. Alberto Vallejo Peña and Sandro Giachi highlight the existence of diverse institutional models of work organization between geographical areas of Europe and compare the situation of four representative countries of southern Europe with that of the rest of Europe. Their conclusions may surprise. Sociology has long been used to highlight the existence of diverse institutional models of work organization between geographical areas of Europe. Based on this, to compare the situation of four representative countries of southern Europe (Spain, Italy, Greece and Portugal) with that of the rest of Europe is proposed. Specifically, through addressing the number of hours worked and the flexibility of working hours as key elements of their institutional model of work organisation, as well as their impact on levels of labour productivity. Taking the model of the varieties of capitalism as a reference, this study compares the behaviour of the Mediterranean (Southern European) countries with other European regions. Indicators have been obtained from the year 2010 and 2015 datasets of the European Work Conditions Survey (EWCS). Including the number of hours worked, the flexibility in the hours of entry and exit, and the tendency to work the same number of hours per day. After comparing averages in both sets and applying linear regressions, the following conclusions have been reached: (1) Productivity in Southern countries is on a par with the European average but far from the more corporatist and liberal (Northern European) areas; (2) the South maintains a high average of hours worked (above the European average) to compensate for the poor productivity of its hours; and (3) the incorporation of flexible schedules is associated with elevated levels of productivity.

In "Similitudes and singularities of higher education systems in the Mediterranean countries: Historical construction, policy and evolution of key indicators", Cláudia Urbano finds that Southern Europe has been presenting many differences with the rest of the continent to ensure comparability of the standards and quality of higher education qualifications. Additionally, Urbano confirms that education in such countries needs strengthening by introducing sustainable development, through a holistic approach, into educational curricula, from primary school to higher education. Knowledge management issues are a high priority topic for growth enhancement in Southern Europe. Thus, we are witnessing a need for a quick and intelligent reaction from organizations to expedite change. Higher education is one of the most important values for changes in societies and exchanges among different societies. Analysing higher education systems in Europe, Southern Europe has been deviating from the rest of the continent, despite the effort of the Bologna Process to ensure comparability in the standards and quality of higher education qualifications. Considering four Southern Europe countries - Portugal, Spain, Italy and Greece - and their link to a certain Mediterranean culture, our proposal is to analyse these countries' higher education systems and their economic state. This can be achieved using indicators on educational stock, economic growth, income distribution, 
supply and demand of higher education and economic indicators relating training and the economy such as graduated employment rates. Also, education public policies will be considered in the analysis as they shape higher education systems' trajectories. Comparing them, we can identify similitudes and singularities in these education systems, leading us to conclude about the existence of a Southern European approach to making higher education a specific value in Mediterranean culture. This topic is even more important as it may be related to the recent focus of EU activities in the Southern Mediterranean region. The Mediterranean Strategy for Sustainable Development (MSSD) recognises that education in the Mediterranean needs strengthening by introducing sustainable development, through a holistic approach, into educational curricula, from primary school to higher education.

Finally, the sixth contribution, "The Mediterranean Diet and the Increasing Demand of the Olive Oil Sector: Shifts and Environmental Consequences", by Bruno Neves and Iva Miranda Pires, tackles one of the most interesting topics of the region's traditions and contributions for social wellbeing, diet. Mediterranean countries play a crucial role as olive oil producers and consumers compared to other world regions. This work focuses on the development of world production, trade and consumption in olive oil. The Mediterranean region stands out from both the rest of the world and the Northern European countries with respect to olive oil production and consumption. Aspects such as communicating the the benefits of a Mediterranean diet, the Slow Food Movement, the International Olive Council campaigns, and the successive Common Agricultural Policies are discussed. Such increases and stimuli brought, and is still bringing, changes to the olive oil sector. Notably, a shifting tendency in production modes as well as modernization of the sector in response to the increasing demand. Such changes are altering landscapes and are considered environmentally harmful to the ecosystems because the production process is shifting to more intensive methods and monoculture plantations.

Much remains to be explored in our research agenda that has not yet been assessed. Our efforts were supported by the interest in this fascinating region, its history, its identities, its languages, its economic structures and fragile sustainable environments, its creative solutions and resilience, as well as the combination of its pluralistic culturalhistorical identity that still is a driving force in a global world. The authors invite colleagues to contribute to the enlargement of this research agenda. Thank you for your interest and attention to a few the of many Southern European challenges.

\section{References}

de Noronha T (2018) Mediterranean and migrations. Journal of Mediterranean Knowledge $3[1]$

de Noronha T, Mangone E (2018) Historical contexts and identities of the mediterranean countries. Journal of Spatial and Organizational Dynamics 6[2]

European Commission (2016) Action Plan on the Integration of Third-Country Nationals. European commission, https://ec.europa.eu/home-affairs/sites/homeaffairs/files/whatwe-do/policies/european-agenda-migration/proposal-implementation-package/docs/20160607/communication_action_plan_integration_third-country_nationals_en.pdf

Vaz E, de Noronha T (2020) Southern Europe - Major Trends and New Prospects. Cities and Nature. Springer Verlag, London (forthcoming)

(7) (5) (c) 2019 by the authors. Licensee: REGION - The Journal of ERSA, European Regional Science Association, Louvain-la-Neuve, Belgium. This article is distributed under the terms and conditions of the Creative Commons Attribution, Non-Commercial (CC BY NC) license (http://creativecommons.org/licenses/by-nc/4.0/). 